\title{
2013 Steele Prizes
}

The 2013 AMS Leroy P. Steele Prizes were presented at the 119th Annual Meeting of the AMS in San Diego, California, in January 2013. The Steele Prizes were awarded to JOHN GUCKENHEIMER and PHILIP HOLMES for Mathematical Exposition, to SAHARON SHELAH for a Seminal Contribution to Research, and to YAKOV SINAI for Lifetime Achievement.

\section{Mathematical Exposition: John Guckenheimer and Philip Holmes}

\section{Citation}

The 2013 Leroy P. Steele Prize for Mathematical Exposition is awarded to John Guckenheimer and Philip Holmes in recognition of their book, Nonlinear Oscillations, Dynamical Systems, and Bifurcations of Vector Fields, Applied Mathematical Sciences, 42, SpringerVerlag, New York, 1983; reprinted with revisions and corrections, 1990.

Dynamical systems underwent a rebirth in the 1960s and 1970s with the work of mathematicians such as (in alphabetical order) Anosov, Arnold, Kolmogorov, Moser, Ruelle, Sinai, Smale, Takens, Thom, and many others on the theoretical side and engineers and experimental physicists such as Lorenz, Swinney, Gollub, and many others on the applied side. Not surprisingly, it was difficult for the two communities to know about each other's work until the publication of the now-classic text by Guckenheimer and Holmes. Thirty years later this book remains in wide use as a standard text for graduate-level courses in mathematics departments and throughout the sciences and engineering, and Chinese and Russian translations have appeared.

In the late 1970 s dynamical systems theory was still largely the preserve of mathematicians, at least in Europe and the Americas (the Soviet Union had maintained somewhat stronger links among mathematical scientists and physicists, chemists, and engineers). Excitement was growing over chaos and sensitive dependence (the butterfly effect) and bifurcation and unfolding theories. Physicists such as Swinney and Gollub were generating experimental data on constrained fluid systems, but the fundamental work of Smale and his students was appearing in journals unknown to many researchers who could most benefit from them, beyond and even within the mathematics community.

Research monographs were beginning to appear: Abraham and Marsden's Foundations of Mechanics (1967) focused on Hamiltonian systems and classical mechanics; Marsden and McCracken edited a collection of papers on Hopf bifurcation (1976). A

DOI: http://dx.doi.org/10.1090/noti972 few research groups were applying center manifold reduction to physical problems, computing normal forms, and unfolding bifurcations, but the Guckenheimer and Holmes work was the first textbook to lay out clearly the theory for dissipative dynamical systems, to separate rigorous results from speculation, to begin to reunite perturbation methods with the geometric and topological ideas of global analysis, and to provide analyses of practical problems.

Both theoretical and applied dynamical systems remain strong research areas, with theoretical research appearing in physics, engineering, and applied mathematics departments and applied work being produced by researchers in mathematics departments - a healthy trend that was given substantial help by the publication of Nonlinear Oscillations, Dynamical Systems, and Bifurcations of Vector Fields.

\section{Biographical Sketches}

John Guckenheimer was born in Baton Rouge, Louisiana, in 1945. He received his undergraduate degree from Harvard in 1966 and his Ph.D. from the University of California Berkeley in 1970 under the direction of Stephen Smale. He held positions at IMPA (1969), the University of Warwick (1969-1970), the Institute for Advanced Study (1970-1972), and the Massachusetts Institute of Technology (1972-1973) before

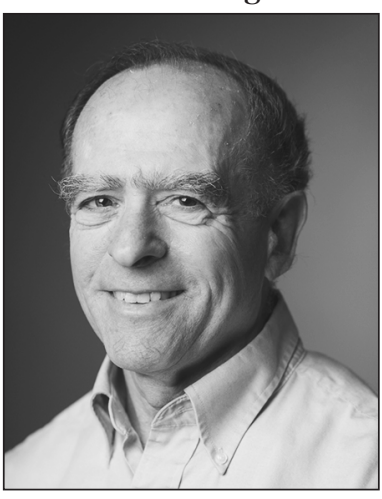

John Guckenheimer joining the faculty of the University of California Santa Cruz (1973-1985). Since 1985 he has been on the faculty of Cornell University, where he is now the A. R. Bullis Professor of Mathematics.

During the past fifteen years, his research has investigated dynamical systems with multiple time scales and associated numerical methods. He has also continued to investigate the use of dynamical systems theory in diverse areas, notably in neuroscience and animal locomotion. He was a 1984 Guggenheim Fellow and is a fellow of the AMS, the American Academy of Arts and Sciences, the American Association for Advancement of Science, and the Society for Industrial and Applied Mathematics (SIAM). He served as president of SIAM in 1997-1998. 


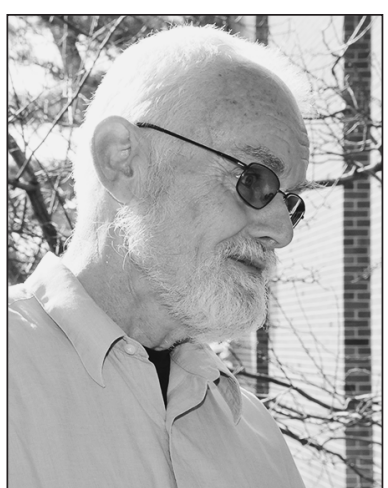

Philip Holmes
Philip Holmes was born in Lincolnshire, England, in 1945 and was educated in engineering science at the Universities of Oxford and Southampton. He taught in the departments of theoretical and applied mechanics and mathematics at Cornell University from 1977 to 1994 . In 1994 he moved to Princeton University, where he is Eugene Higgins Professor of Mechanical and Aerospace Engineering, professor of applied and computational mathematics, associated faculty in the Department of Mathematics, and a member of Princeton's Neuroscience Institute. Much of his research has been in dynamical systems and their applications in engineering and the physical sciences, but in the past fifteen years he has increasingly turned to biology. He currently works on the neuromechanics of animal locomotion and neurodynamics of decision making. He is a member of the American Academy of Arts and Sciences, an honorary member of the Hungarian Academy of Sciences, and a fellow of the AMS, of the American Physical Society, and of SIAM. He has also published four collections of poems (Anvil Press, London).

\section{Joint Response from John Guckenheimer and Philip Holmes}

We are honored and delighted to receive the Steele Prize for Mathematical Exposition. We come from very different places (Baton Rouge, Louisiana, and Brigg, Lincolnshire, United Kingdom) and very different training: John from Ph.D. studies with Steve Smale and Phil from applied mechanics. We first met in 1976 at a dynamical systems conference in Southampton coorganized by David Rand. At that time rapid advances in dynamical systems theory were stimulating experimental work that demonstrated the usefulness of the theory in explaining empirical phenomena across the sciences and engineering. We saw a real need for a book that made the new mathematics accessible to a broad audience, including mature scientists and students. The excitement of the period was captured vividly by James Gleick in his book, Chaos: Making a New Science, Penguin Books, New York, 1987, which received the first JPBM Communications Award in 1988.

In the late 1970s Phil and David Rand, after working together on nonlinear oscillators, began teaching courses in dynamical systems and assembling notes toward a book. Independently, John also began planning a book. In spring 1981
Phil visited UC Berkeley and David visited UC Santa Cruz. We discussed continuing the writing as a three-author team, but ultimately David decided to withdraw while the two of us proceeded. We completed the first draft in approximately nine months, starting with notes for applied dynamical systems courses that we taught at UC Santa Cruz and Cornell and spending an hour or two discussing our differences on the phone every Friday. We received enthusiastic support from Jerry Marsden and others. Walter Kaufmann-Buehler at SpringerVerlag was willing to take a risk in pricing the book at a level that would encourage individuals to buy it. Our handwritten manuscript was painstakingly typed by Dolores Pendell at Cornell's Center for Applied Mathematics, and the diagrams were produced by Barbara Boettcher.

The book's success has been extraordinarily gratifying, especially when younger (than us!) scientists tell us that they studied it carefully and keep returning to take it from their bookshelves. We tried hard to explain mathematical concepts and arguments in their simplest manifestations while relying on as little formal training as seemed feasible. It helped that we came to the interface between mathematics and the physical sciences from opposite sides. With our different backgrounds, we sought to bring alive how dynamical systems theory has been enriched repeatedly by questions from the "real world", while, at the same time, demonstrating the power of mathematical thinking and abstraction to unify the sciences. We thank the Committee for recognizing our efforts to present significant results to a broad scientific audience that stretches far beyond the boundaries of mathematics.

\section{Seminal Contribution to Research: Saharon Shelah}

\section{Citation}

The 2013 Leroy P. Steele Prize for Seminal Contribution to Research is awarded to Saharon Shelah for his book Classification Theory and the Number of Nonisomorphic Models, Studies in Logic and the Foundations of Mathematics, 92, North-Holland Publishing Co., Amsterdam-New York, 1978; 2nd edition, 1990.

Before Shelah's work, the great theorem of pure model theory was Morley's theorem on categoricity. It concerned a class of theories whose uncountable models are completely determined by their cardinality. Shelah visualized a vast extension of the

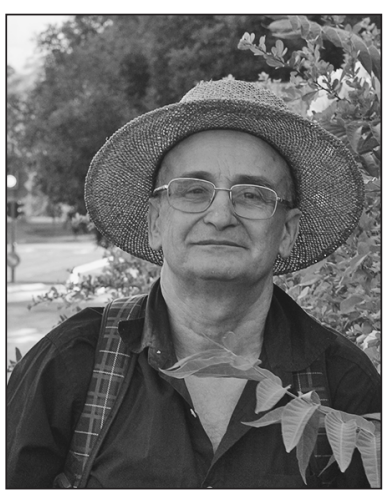

Saharon Shelah 
problem to a classification of arbitrary first-order theories, based on the number of models they may have of a given uncountable size. Solving this problem required some twenty years that made model theory into a mature field, completely transforming its aims, methods, and ability to connect to algebra and geometry.

Shelah isolated the class of stable theories, where finitely generated extensions admit, in a certain local sense, finitary descriptions. He was able to show, on the other hand, that any unstable theory has the maximum set-theoretically permissible number of models. All theories of modules are stable. Among the stable theories, he isolated the superstable theories, analogous to Noetherian rings, and again found many models if this condition fails. These were the first two of a series of dividing lines, characterized by a deep theorem on either side. On the stable side, he was able to define a canonical tensor product of extensions of structures and made it into an incisive tool for the decomposition of structures. An arsenal of notions became available to the previously bare-handed model theorist: algebraic closure, canonical bases, imaginary sorts, domination, forking, regular types. These concepts proved useful beyond the stable framework and led to substantial applications when investigated in algebraic settings. The problem of the number of models was solved in the second edition of his monograph, but the ideas of the solution remained central and proved critical for many others. It would be impossible to imagine model theory today without them.

\section{Biographical Sketch}

Saharon Shelah earned his B.Sc. from Tel Aviv University, his M.Sc. from the Hebrew University under the supervision of Professor H. Gaifman, and his Ph.D. from the Hebrew University under the supervision of Professor M. Rabin. He has taught at the Hebrew University and Rutgers University, among others. He is a member of the Israel Academy of Sciences and Humanities and the American Academy of Arts and Sciences.

\section{Response}

I am grateful for this great honour.

While it is great to find full understanding of that for which we have considerable knowledge, I have been attracted to trying to find some order in the darkness; more specifically, finding meaningful dividing lines among general families of structures. This means that there are meaningful things to be said on both sides of the divide: characteristically, understanding the tame ones and giving evidence of being complicated for the chaotic ones. It is expected that this will eventually help in understanding even specific classes and even specific structures. Some others see this as the aim of model theory; not so for me. Still I expect and welcome such applications and interactions. It is a happy day for me that this line of thought has received such honourable recognition. Thank you.

\section{Lifetime Achievement: Yakov Sinai}

\section{Citation}

The 2013 Steele Prize for Lifetime Achievement is awarded to Yakov Sinai for his pivotal role in shaping the theory of dynamical systems and for his groundbreaking contributions to ergodic theory, probability theory, statistical mechanics, and mathematical physics.

Sinai's research exhibits a unique combination of brilliant analytic technique, outstanding geometric intuition, and profound understanding of underlying physical phenomena. His work highlights deep and unexpected connections between dynamical systems and statistical mechanics.

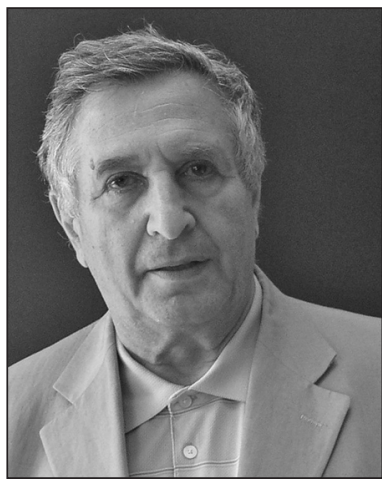
Sinai has opened up new directions, including Kolmogorov-Sinai entropy, Markov partitions, and Sinai-Ruelle-Bowen measures in the hyperbolic theory of dynamical systems; dispersing billiards, a rigorous theory of phase transitions in statistical mechanics and space-time chaos. In addition, Sinai has made seminal contributions in the theory of Schrödinger operators with quasi-periodic potentials, random walks in random environments, renormalization theory, and statistical hydrodynamics for Burgers and Navier-Stokes equations.

Sinai pioneered the study of dispersing billiards: dynamical systems which model the motion of molecules in a gas. The simplest example of such a billiard table, a square with a disk removed from its center, is called "Sinai's billiard". Studying billiard motions within the framework of hyperbolic theory, Sinai discovered that they exhibit deep ergodic and statistical properties (such as the central limit theorem). Owing to Sinai's work, some key laws of statistical mechanics for the Lorentz gas can be established with mathematical rigor. In particular, Sinai made the first steps towards justification of Boltzmann's famous ergodic hypothesis, proposed in the end of the nineteenth century: "For large systems of interacting particles in equilibrium, time averages are close to the ensemble average." Sinai returned to this subject several times in the period 1970-1990 with various coauthors, including his students Bunimovich and Chernov.

Together with his student Pirogov, Sinai created a general theory of low-temperature phase transitions for statistical mechanics systems with a finite 
number of ground states. Pirogov-Sinai theory forms essentially the basis for modern equilibrium statistical mechanics in a low-temperature regime.

Sinai made seminal contributions to the theory of random walks in a random environment. With his model, known nowadays as "Sinai's random walk", he obtained remarkable results about its asymptotic behavior. With his student Khanin, Sinai pioneered applications of the renormalization group method to multifractal analysis of the Feigenbaum attractor and to the KolmogorovArnold-Moser theory on invariant tori of Hamiltonian systems.

In the past fifteen years Sinai has brought novel tools and insights from dynamical systems and mathematical physics to statistical hydrodynamics, obtaining new results for the Navier-Stokes systems. Specifically, along with D. Li, Sinai devised a new renormalization scheme which allows the proof of existence of finite time singularities for complex solutions of the Navier-Stokes system in dimension three.

Sinai's mathematical influence is overwhelming. During the past half-century he has written more than 250 research papers and a number of books. Sinai's famous monograph, Ergodic Theory (with Cornfeld and Fomin), has been an introduction to the subject for several generations, and it remains a classic.

Sinai supervised more than fifty Ph.D. students, many of whom have become leaders in their own right. Sinai's work is impressive for its breadth. In addition to its long-lasting impact on pure mathematics, it has played a crucial role in the creation of a concept of dynamical chaos which has been extremely important for the development of physics and nonlinear science over the past thirty-five years. The Steele Prize for Lifetime Achievement is awarded to Sinai in recognition of all these achievements.

\section{Biographical Sketch}

Yakov G. Sinai was born in 1935 in Moscow, Soviet Union, now Russia. He received his Ph.D. degree (called a Candidate of Science in Russia) and then his doctorate degree (Doctor of Science) from Moscow State University. For several years, he held combined positions at Moscow State University and the Landau Institute of Theoretical Physics of the Russian Academy of Sciences. Since 1993, he has been a professor in the mathematics department of Princeton University.

Sinai has received various honors recognizing his contributions. He was elected as a foreign associate of the National Academy of Sciences and a foreign member of the Academy of Arts and Sciences. He is a full member of the Russian Academy of Sciences, and he was recently elected as a foreign member of the Royal Society in London. He is also a member of the Brazilian Academy of
Science, the Hungarian Academy of Science, the Polish Academy of Science, and Academia Europea.

Among his other recognitions are the Wolf Prize in Mathematics, the Nemmers Prize, the Lagrange Prize, the Boltzmann Medal, the Dirac Medal, and the Poincaré Prize.

\section{Response}

It is a great honor to be awarded the Steele Prize for Lifetime Achievement from the American Mathematical Society. I worked in several directions in mathematics, including the theory of dynamical systems, statistical and mathematical physics, and probability theory.

My mentors who had a big influence on me were A. N. Kolmogorov, V. A. Rokhlin, and E. B. Dynkin. I also benefited a lot from many contacts with my colleagues. I was very fortunate to have talented students, many of whom became strong and famous mathematicians. Unfortunately, it is not possible to list the names of all of them here. I thank my family and friends for their encouragement and support. Finally, I thank the selection committee for its work.

\section{About the Prize}

The Steele Prizes were established in 1970 in honor of George David Birkhoff, William Fogg Osgood, and William Caspar Graustein. Osgood was president of the AMS during 1905-1906, and Birkhoff served in that capacity during 1925-1926. The prizes are endowed under the terms of a bequest from Leroy P. Steele. Up to three prizes are awarded each year in the following categories: (1) Lifetime Achievement: for the cumulative influence of the total mathematical work of the recipient, high level of research over a period of time, particular influence on the development of a field, and influence on mathematics through Ph.D. students; (2) Mathematical Exposition: for a book or substantial survey or expository research paper; (3) Seminal Contribution to Research: for a paper, whether recent or not, that has proved to be of fundamental or lasting importance in its field or a model of important research. Each Steele Prize carries a cash award of US\$5,000.

Beginning with the 1994 prize, there has been a five-year cycle of fields for the Seminal Contribution to Research Award. For the 2013 prize, the field was logic. The Steele Prizes are awarded by the AMS Council acting on the recommendation of a selection committee. For the 2013 prizes, the members of the selection committee were Yakov Eliashberg, John E. Fornæss, Irene M. Gamba, Barbara L. Keyfitz, Tomasz S. Mrowka, Gang Tian, Akshay Venkatesh, Lai-Sang Young, and Efim I. Zelmanov. The list of previous recipients of the Steele Prize may be found on the AMS website at http://www.ams .org/prizes-awards.

-Elaine Kehoe 